\title{
Effect of BLUP prediction on genomic selection: practical considerations to achieve greater accuracy in genomic selection
}

\author{
Patricio Munoz ${ }^{1 *}$, Marcio Resende ${ }^{2}$, Gary Peter ${ }^{3}$, Dudley Huber ${ }^{4}$, Matias Kirst ${ }^{3}$, Tania Quesada ${ }^{4}$ \\ From IUFRO Tree Biotechnology Conference 2011: From Genomes to Integration and Delivery \\ Arraial d Ajuda, Bahia, Brazil. 26 June - 2 July 2011
}

\section{Background}

Prediction of breeding values (BV) using only genotypic information is the final goal of Genomic Selection (GS) [1]. Commonly, BV prediction from traditional BLUP analysis is the input for constructing GS prediction models, and GS predicted BVs are correlated with traditional BLUP BVs to estimate the accuracy of GS models. The use of GS in plant breeding depends on the accuracy of the GS models to predict the BVs. Therefore, better accuracy and less bias in traditional BLUP BVs should improve the final accuracy of GS predictions. Such improvements in GS predictions are not due to GS modeling itself, but rather to the reduced noise in the BLUP BV used as input.

Improvements in BLUP BV can be obtained simply by correcting errors in the pedigree [2] or using more complex approaches, such as applying a realized relationship matrix (RRM) in the BLUP prediction as an alternative to the relationship matrix (A) based on expected values derived from the pedigree [3]. Misspecification of effects in BLUP models tends to produce upward bias in the BV estimates, which also impact GS accuracy [4]. In addition, not correcting with the additive-genetic relationship information in the GS prediction model leads to overestimates in accuracies due to inadequate accounting for confounding genetic relationships found in the training population [5]. The inflated accuracy cannot be exploited in future generations and should be guarded against.

Our objective was to use real data to study the effect on the GS accuracy from 1) pedigree errors, 2)

\footnotetext{
* Correspondence: p.munoz@ufl.edu

'Plant Molecular and Cellular Biology Program, University of Florida, Gainesville, FL, USA

Full list of author information is available at the end of the article
}

incorporation of the RRM in the BLUP analysis, 3) misspecification of non-additive effects in the BLUP analysis and 4) the effect of ignoring the additive-genetic relationship in the GS prediction model.

\section{Methods}

Height (HT) was measured in one field test containing 860 clonally propagated loblolly pine trees $(\sim 8$ ramets per genotype) derived from 32 parents crossed in a circular mating design. The population was genotyped using the Illumina Infinium ${ }^{\mathrm{TM}}$ assay (Illumina, San Diego, CA) with 7,216 SNPs. A total of 3,938 SNPs were selected for use in GS based on frequency of polymorphism across genotypes, quality and reliability of the reads. SNP markers were used to estimate the RRM following a recently published method [3] where identity by descent is determined relative to a base population. RRM values were adjusted as recommended [6] to obtain less biased variance estimations. Based on the RRM, a new pedigree was constructed.

Several BLUP models were fit in ASReml to study the following effects:

Model 1: Additive + non-additive effects model original pedigree

Model 2: Additive + non-additive effects model - new pedigree (expected A matrix)

Model 3: Additive model - new pedigree based (expected A matrix)

Model 4: Additive model - RRM (observed A matrix)

The BVs obtained from models 1-4 were deregressed and used to construct GS prediction models with GBLUP [1]. Additionally, two GS prediction models were constructed based on the raw BVs (not deregressed) from models 3 and 4 to study the effect of 


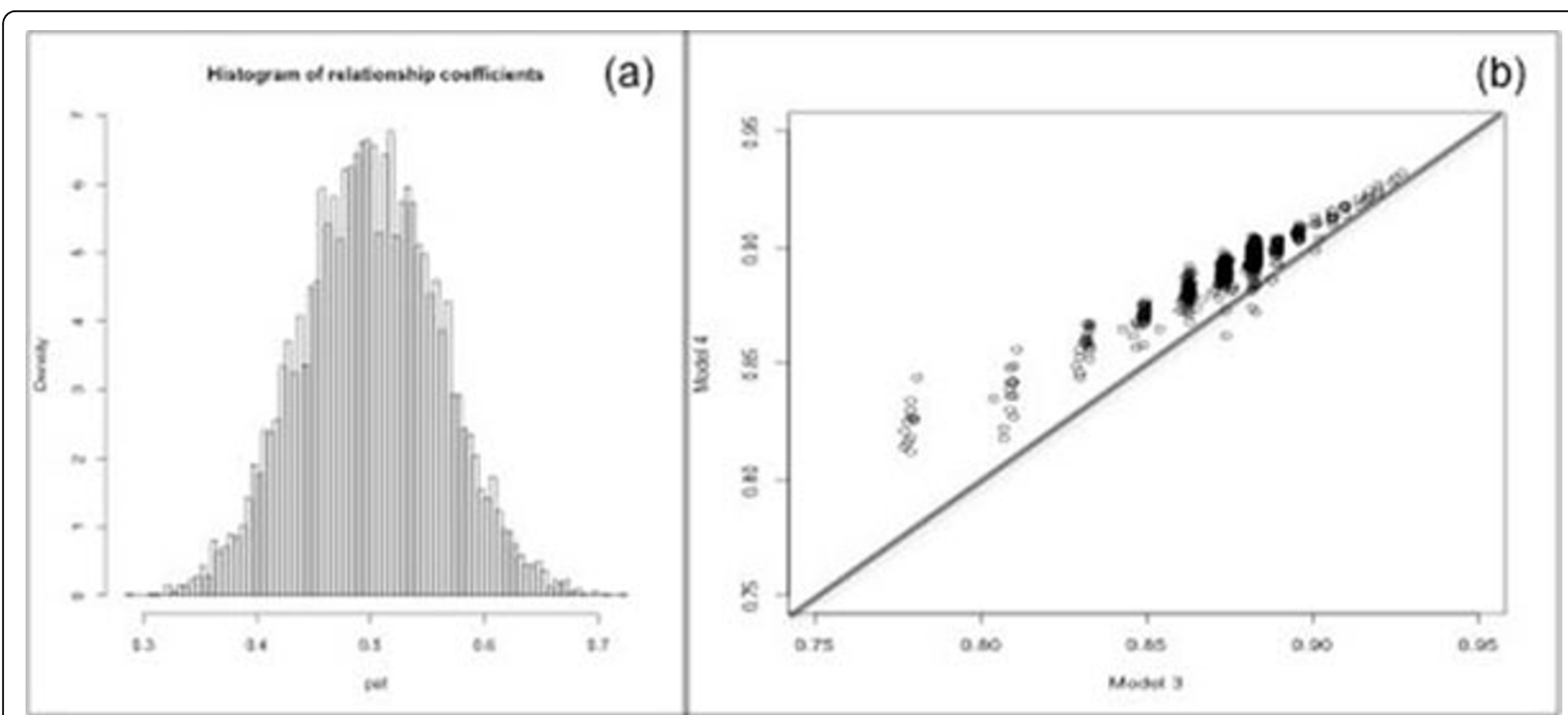

Figure 1 (a) Distribution of relationship values around the expected mean (0.5) for full-sib individuals $n=6475$, SD=0.06; (b) Accuracy of BLUP prediction in pedigree-based (Model 3) and RRM-based (Model 4) analysis

ignoring the additive-genetic relationships in the training population when constructing the GS model.

\section{Results and discussion}

The RRM among 6475 full-sib pairs (Figure 1a) showed a normal distribution of relationship coefficients around the expected value.

As expected, when the RRM was used to correct the original pedigree [3] the accuracy of the BLUP predictions increased from 0.80 to 0.85 (Table 1), and GS accuracy improved from 0.64 to 0.77 [4]. When the RRM was used directly, instead of the corrected pedigree accuracy of the BLUP, the BVs improved (Figure 1b). Improved BLUP BV estimates also resulted in the improvement of the accuracy of GS predictions from 0.58 to 0.60 . The same results were obtained when the additive model was compared with the full model, indicating that misspecification of effects in the BLUP model will cause a decrease in the GS accuracy [5]. In addition, as shown [6] ignoring the additive-genetic relationship dramatically inflates GS

Table 1 Heritability, BLUP and GS accuracy for models 1, 2, 3 and 4.

\begin{tabular}{ccccc}
\hline ModelNumber & Heritability $^{1}$ & BLUP $^{2}$ Accuracy $^{2}$ & Deregressed & GS $^{3}$ Accuracy $^{3}$ \\
\hline 1 & 0.26 & 0.80 & Yes & 0.64 \\
2 & 0.31 & 0.85 & Yes & 0.77 \\
3 & 0.34 & 0.87 & Yes & 0.58 \\
3 & 0.34 & 0.87 & No & 0.87 \\
4 & 0.36 & 0.89 & Yes & 0.60 \\
4 & 0.36 & 0.89 & No & 0.88 \\
\hline
\end{tabular}

\footnotetext{
${ }^{1}$ Narrow sense heritability, ${ }^{2}$ Average of accuracy among all clones, ${ }^{3}$ correlations between BLUP BVs and GS-BVs prediction
}

accuracy from 0.58 to 0.87 and from 0.60 to 0.88 for Models 3 and 4 respectively.

\section{Conclusions}

To maximize the true accuracy of GS, it is recommended: 1) construct a RRM for the training population that should be used to correct the pedigree and to predict the BLUP BVs, 2) correct for non-additive effects if using a family related training population, and 3) deregress BVs prior to use as input for construction of GS prediction models.

\section{Author details}

'Plant Molecular and Cellular Biology Program, University of Florida, Gainesville, FL, USA. ${ }^{2}$ Genetic and Genomics Graduate Program, University of Florida, Gainesville, FL, USA. ${ }^{3}$ School of Forestry Resources and Conservation, Genetic and Genomics Graduate Program, Plant Molecular and Cellular Biology Program, University of Florida, Gainesville, FL, USA. ${ }^{4}$ School of Forestry Resources and Conservation, University of Florida, Gainesville, FL, USA.

Published: 13 September 2011

\section{References}

1. Meuwissen T, Hayes B, Goddard M: Prediction of total genetic value using genome-wide dense marker maps. Genetics 2001, 157:1819-1829.

2. Sander K, Bennewitz J, Kalm E: Wrong and missing sire information affects genetics gain in the angeln dairy cattle population. J Dairy Sci 2006, 89:315-321.

3. Powell J, Visscher P, Goddard M: Reconciling the analysis of IBD and IBS in complex trait studies. Nat Rev Genet 2010, 11:800-805.

4. Lee $S$, Goddard M, Visscher $P$, van der Werf J: Using the realized relationship matrix to disentangle confounding factors for the estimation of genetic variance components of complex traits. Genet Sel Evol 2010, 42:22.

5. Habier D, Fernando R, Dekkers J: The impact of genetic relationship information on genomic-assisted breeding values. Genetics 2007, 42:5. 
6. Yang J, Benyamin B, McEvoy B, Gordon S, Henders A, Nyholt D, Madden P, Heath A, Martin N, Montgomery G, Goddard M, Visscher P: Common SNPs explain a large proportion of the heritability for human height. Nat Genet 2010, 42:565-571.

doi:10.1186/1753-6561-5-S7-P49

Cite this article as: Munoz et al:: Effect of BLUP prediction on genomic selection: practical considerations to achieve greater accuracy in genomic selection. BMC Proceedings 2011 5(Suppl 7):P49.

Submit your next manuscript to BioMed Central and take full advantage of:

- Convenient online submission

- Thorough peer review

- No space constraints or color figure charges

- Immediate publication on acceptance

- Inclusion in PubMed, CAS, Scopus and Google Scholar

- Research which is freely available for redistribution

Submit your manuscript at www.biomedcentral.com/submit 